\title{
Multi-frequency VSOP and VLBI observations of the quasar $3 \mathrm{C} 309.1$
}

\section{Marcin P. Gawroński*}

Toruń Centre for Astronomy, Nicolaus Copernicus University, Poland

E-mail: motylek@astro.uni.torun.pl

\section{Andrzej J. Kus}

Toruń Centre for Astronomy, Nicolaus Copernicus University, Poland

E-mail: ajkeastro.uni.torun.pl

\begin{abstract}
We present multi-frequency observational results for the Compact Steep-Spectrum (CSS) quasar 3C 309.1. The observations were carried out with the VSOP at 1.6 and $4.8 \mathrm{GHz}$ and the VLBA at $15.4 \mathrm{GHz}$. The source has a distorted, one-sided radio jet. Relativistics effects and physical properties are discussed. Comparing the predicted and observed X-ray fluxes in the framework of the synchrotron self-Compton model we derive the beaming factor for 3C 309.1. The complex structure of the jet may be explained by a relativistic helical flow in a heterogeneous, clumpy ISM.
\end{abstract}

The 8th European VLBI Network Symposium

September 26-29, 2006

Toruń, Poland

\footnotetext{
* Speaker.
} 


\section{Introduction}

The quasar 3C 309.1 belongs to the class of Compact Steep-Spectrum (CSS) radio sources, which includes objects with projected linear sizes $<20 \mathrm{kpc}$ and steep radio spectra $(\alpha>0.5, S \propto$ $v^{-\alpha}$ ) [7, 13]. Their fraction is high - up to $30 \%$ depending on the selection frequency. CSS sources are identified with quasars, radio galaxies and Seyferts and the morphological separation between CSS quasars and galaxies - similar to that observed in the case of larger sources - has been found: radio galaxies have simple double radio structures, whereas quasars show either triple structures

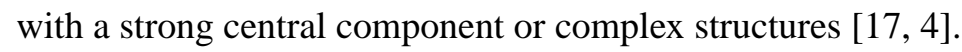

$3 \mathrm{C} 309.1$ is one of the most luminous object in the class $\left(L_{178 \mathrm{MHz}}=8 \times 10^{28} \mathrm{~W} \mathrm{~Hz}^{-1}\right)$ and its radio structure has been studied with the VLA, MERLIN and the VLBI [9, 10, 1, 12]. The overall extent of the source is $2 . " 2$ which translates to $80 \mathrm{kpc}^{1}$ for the redshift $z=0.905$ [3]. This quasar appears as a triple source in the VLA and MERLIN images. There is a dominant central compact region, a curved, one-sided, 1 arcsecond-long jet emerging to the east with the hot-spot at the end and a low surface brightness component with extended north-south structure.

\section{Observations}

We observed 3C 309.1 with HALCA at $1.6 \mathrm{GHz}$ on 19 December 1997 and at $4.8 \mathrm{GHz}$ on 11 May 1998. We used all VLBA stations in both experiments with the addition of Goldstone $70 \mathrm{~m}$ radio telescope at $1.6 \mathrm{GHz}$ and two EVN stations (Effelsberg and Toruń) at $4.8 \mathrm{GHz}$. The data from HALCA satellite were received by the tracking stations at Goldstone, Tidbinbilla and Robledo.

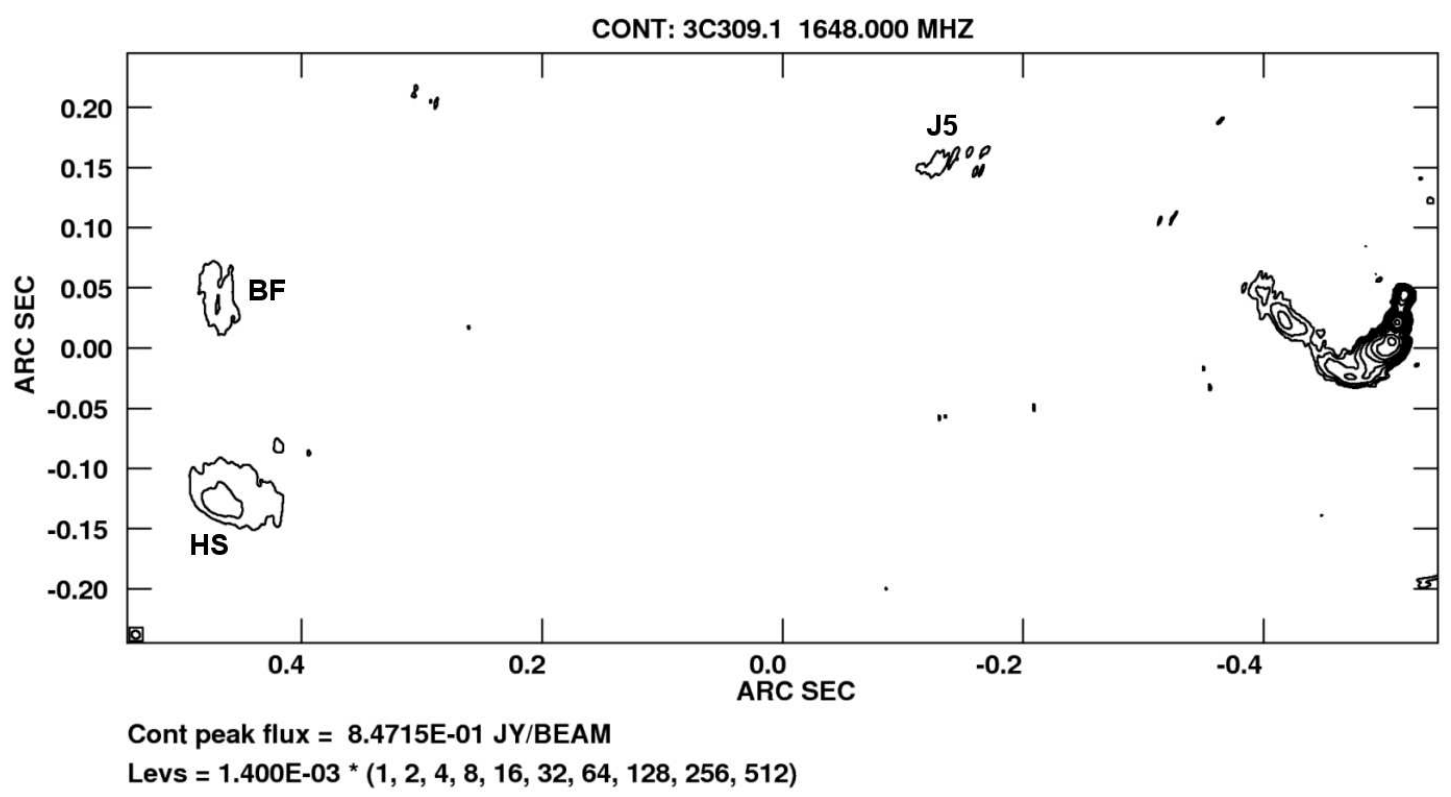

Figure 1: Large-scale VLBI map of 3C 309.1 at $1.6 \mathrm{GHz}$.

\footnotetext{
${ }^{1}$ We assume a cosmology with $H_{0}=75 \mathrm{~km} \mathrm{~s}^{-1} \mathrm{Mpc}^{-1} \Omega_{M}=0.3, \Omega_{\Lambda}=0.7$
} 


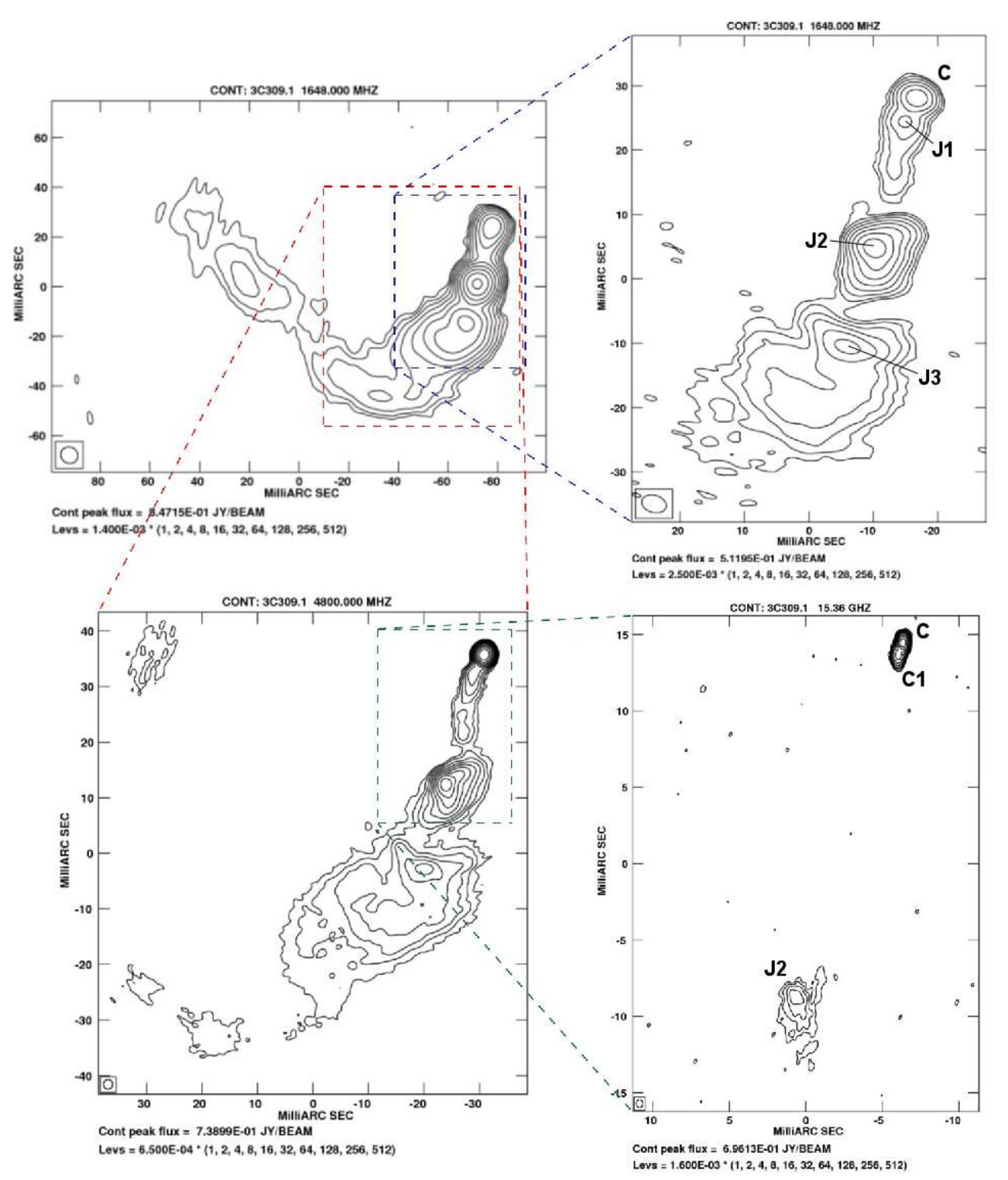

Figure 2: The composition of VLBI and VSOP maps of 3C 309.1. Upper left: the inner part of the jet at 1.6 GHz. Upper right: the VSOP map at $1.6 \mathrm{GHz}$. Lower left: the VLBI map at $4.8 \mathrm{GHz}$. Lower right: the VLBI map at $15.4 \mathrm{GHz}$. The main components are marked in the 1.6-GHz map. The superluminal feature $\mathrm{C} 1$ is marked in the VLBI 15.4-GHz map.

Clear fringes were found to HALCA at both epochs. We also carried out the VLBA observation at 15.4 GHz on 1 September 2002. The data were globally fringe fitted and calibrated in a standard way with AIPS. For the experiments including HALCA, we produced images for both: all the data and ground-based baselines only. The resulting images of 3C 309.1 are presented in Fig.1 and Fig. 目. We have labelled four main components of the jet as C (core), J1, J2 and J3.

The ground-based map at $1.6 \mathrm{GHz}$ (Fig.11) shows a complex jet structure and previously undetected features at some distance from the core C. The J5 component $\left(0 .{ }^{\prime \prime} 4\right.$ from the core at 


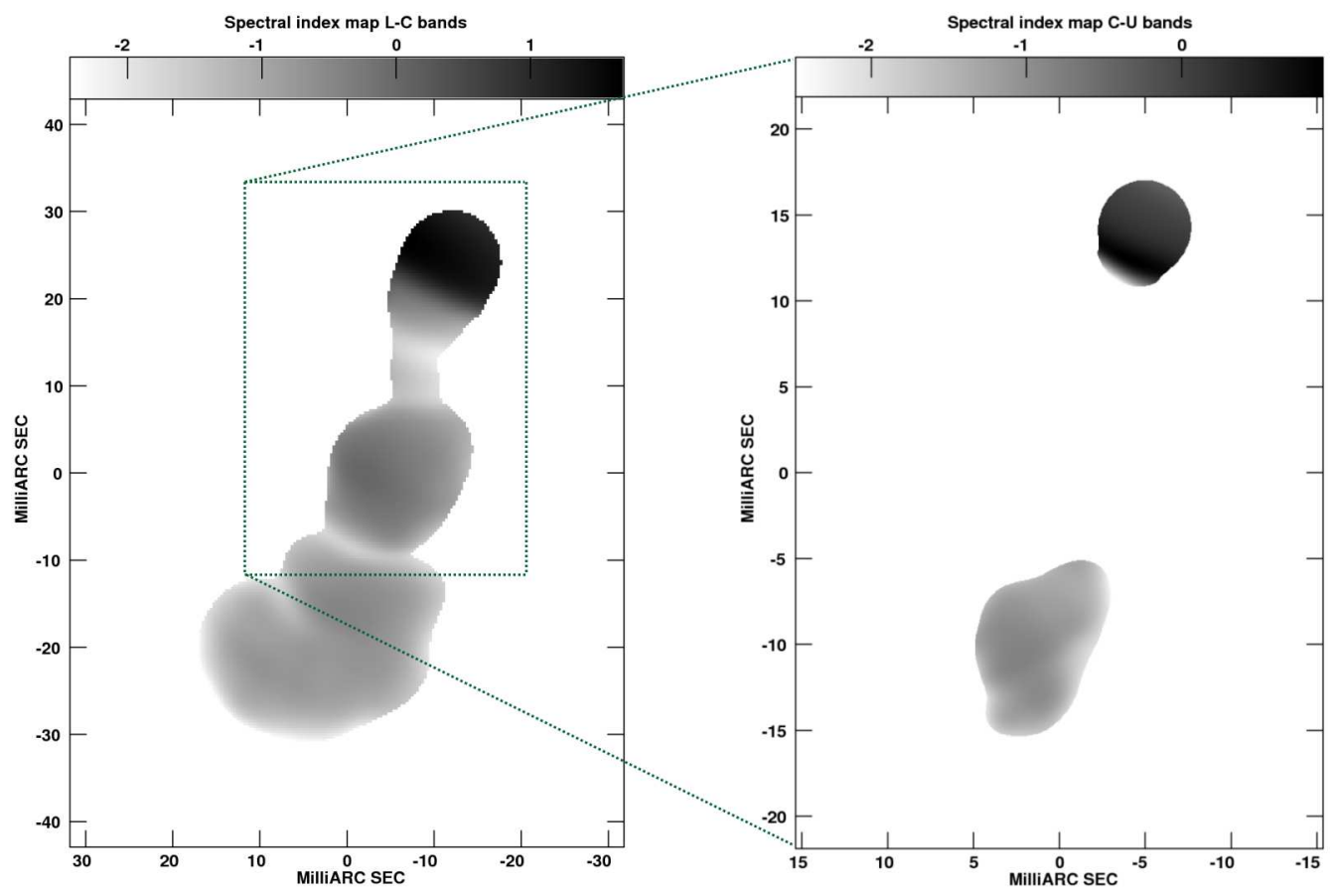

Figure 3: The spectral index maps. Left: between 1.6 and 4.8 GHz. Right: between 4.8 and $15.4 \mathrm{GHz}$.

$\left.\mathrm{P} . \mathrm{A} \simeq-75^{\circ}\right)$ is connected with the bend of the jet visible on the MERLIN maps [12] whereas HS $\left(0{ }^{\prime \prime} 98\right.$ at $\left.\mathrm{P} . \mathrm{A} \simeq-90^{\circ}\right)$ and $\mathrm{BF}\left(1^{\prime \prime}\right.$ at $\left.\mathrm{P} . \mathrm{A} \simeq-100^{\circ}\right)$ are probably a hot-spot and a backflow, respectively, similar to those observed in the standard FR II radio lobes. The inner part of the jet is dominated by components $\mathrm{C}$ and $\mathrm{J} 2$, which have similar brightness at $4.6 \mathrm{GHz}$ and are connected by a smoothly curving jet. There is a bright spot $\mathrm{J} 1$ of emission inside this part of the jet. Components $\mathrm{J} 2$ and $\mathrm{J} 3$ are embedded in large regions of emission also detected in previous observations [1]. The jet extends about 80 mas at $\mathrm{P} . \mathrm{A} \simeq-155^{\circ}$, rapidly changes its direction to $\mathrm{P} . \mathrm{A} \simeq-45^{\circ}$ and extends another 110 mas as seen on the ground-based VLBI map at $1.6 \mathrm{GHz}$ (Fig.1).

\section{Results}

The maps of the spectral index between 1.6 and $4.8 \mathrm{GHz}$, and between 4.8 and $15.4 \mathrm{GHz}$ are shown in Fig.3. We noticed that the flattening of spectral index of the jet is connected with the increase of brightness. This could be an artifact introduced during the reduction process but a physical connection with the change of the jet direction is also possible. If we assume a stable helical path model [10] this effect could easily be explained by the increased beaming factor and supports the idea of a stable jet path. Components $\mathrm{J} 2$ and $\mathrm{J} 3$ are those regions of the flow where the local velocity vector is close to the line of sight. Theoretical studies of Kelvin-Helmholtz instabilities in the relativistic jets have shown that they are able to create stable helical pattern [6]. It has been suggested [15] that the origin of the infrared radiation is in the inner $\sim 250 \mathrm{pc}$, thus the helical part of the 3C 309.1 jet is placed in a very dense medium. We discovered a relativistic motion of the new blob $\mathrm{C} 1$ by comparing our map obtained at $15.4 \mathrm{GHz}$ with the MOJAVE project data 


\begin{tabular}{c|cc}
\hline \hline Component & $T_{B}[\mathrm{~K}](1)$ & $F_{t h} / F_{X}$ \\
\hline \hline $\mathrm{C}$ & $2.6 \times 10^{11}$ & 10 \\
$\mathrm{~J} 1$ & $7.6 \times 10^{8}$ & $<10^{-5}$ \\
$\mathrm{~J} 2$ & $1.8 \times 10^{10}$ & $<10^{-5}$ \\
$\mathrm{~J} 3$ & $7.9 \times 10^{8}$ & $<10^{-5}$ \\
\hline \hline
\end{tabular}

Table 1: The brightness temperature and the ratio of predicted to observed X-ray flux for main components. Note: (1) We used maps at $4.8 \mathrm{GHz}$.

[8, 11], and found $\mathrm{C} 1$ apparent angular separation rate $\mu=0.15 \pm 0.02 \mathrm{mas} / \mathrm{yr}$ which corresponds to $\beta_{\text {app }}=7.0 \pm 0.5 \mathrm{c}$. Such a behaviour was already suggested in [10] and [14]. No change in the position of component $\mathbf{J} 2$ relative to the core was detected. We derived the Doppler factor using the estimated synchrotron self-Compton X-ray flux. The self-Compton flux can be predicted based on the angular size of the components $\theta_{r}$, the synchrotron flux $F_{r}$ at frequency $v_{r}$, and the Doppler factor $\delta$. By comparing the observed and the predicted self-Compton fluxes one can derive $\delta$ in the case of moving sphere according to the formula [5]:

$$
\delta=f(\alpha) F_{r}\left[\frac{\ln \left(v_{b} / v_{r}\right)}{F_{x} \theta_{r}^{6+4 \alpha} v_{x}^{\alpha} v_{r}^{5+3 \alpha}}\right]^{1 /(4+2 \alpha)}(1+z)
$$

where $F_{x}$ and $v_{x}$ are $\mathrm{X}$-ray flux and frequency, $v_{b}$ is the synchrotron high frequency cut-off (assumed to be $\left.10^{14} \mathrm{~Hz}\right), \alpha$ is the spectral index of optically thin synchrotron emission and $f(\alpha) \simeq 0.08 \alpha+$ 0.14 . Knowing the above, we estimated the Lorentz factor $\gamma$ which corresponds to the bulk velocity of the emitting plasma $\gamma$, and the angle $\phi$ between the line of sight and the direction of the bulk velocity $(\gamma=1.5, \phi=15.5)$. To evaluate these, we used the data from the Chandra archive, $F_{2-10 \mathrm{keV}}=9.83 \times 10^{-8} \mathrm{Jy}$ obtained on 28 January 2002 [16]. As the epochs of the Chandra and the VLBA observations at $15.4 \mathrm{GHz}$ were close, we choose these to calculate the ratios of the predicted to the observed X-ray flux for each main component. They are presented in Tab 1 along with the brightness temperatures $T_{b}$ derived from $4.8 \mathrm{GHz}$ maps. If we assume that the orientation of the central engine is similar to that of extended structures visible in the VLA and MERLIN maps then the overall size of 3C 309.1 would be $\sim 80 \mathrm{kpc}$. Thus, it is plausible to assume that 3C 309.1 is a small FR II radio galaxy oriented close to the line of sight and is similar to 3C 216 [2].

We gratefully acknowledge the VSOP Project, which is led by the Institute of Space and Astronautical Science of Japan Aerospace Exploration Agency, in cooperation with many organizations and radio telescopes around the world. This work has made use of the VLBA, which is an instrument of the National Radio Astronomy Observatory, a facility of the National Science Foundation, operated under cooperative agreement by Associated Universities, Inc. The European VLBI Network is a joint facility of European, Chinese and South African radio astronomy institutes funded by their national research councils. We are grateful to the group of the VLBA 2-cm Survey and the group of the MOJAVE project for providing their data. 


\section{References}

[1] S. E. Aaron, J. F. C. Wardle, D. H. Roberts, Vistas Astron. 41, 225 (1997).

[2] P. D. Barthel, T. J. Pearson, A. C. S. Readhead, ApJ 329, 51 (1998).

[3] G. R. Burbidge, E. M. Burbidge, Nature 222, 735 (1969).

[4] R. Fanti, C. Fanti, R. T. Schilizzi et al., A\&A 231, 333 (1990).

[5] G. Ghisellini, P. Padovani, A. Celotti, L. Maraschi, ApJ 407, 65 (1993).

[6] P. E. Hardee, ApJ 318, 78 (1987).

[7] V. K. Kapahi, A\&AS 43, 381 (1981).

[8] K. I. Kellermann, M. L. Lister, D. C. Homan et al., ApJ 609, 539 (2004).

[9] A. J. Kus, P. N. Wilkinson, R. S. Booth, MNRAS 194, 527 (1981).

[10] A. J. Kus, P. N. Wilkinson, T. J. Pearson,, A. C. S. Readhead in proceedings of Parsec-scale radio jets, p.161 (Cambridge University Press, Cambridge) (1990).

[11] M. L. Lister, D. C. Homan, AJ 130, 1389 (2005).

[12] E. Lüdke, S. T. Garrington, R. E. Spencer et al., MNRAS 299, 467 (1998).

[13] J. A. Peacock, J. V. Wall, MNRAS 198, 843 (1982).

[14] E. Ros, Extending and exploring the $2 \mathrm{~cm}$ Survey sample in Proceedings of the 7th European VLBI Network Symposium(2004).

[15] R. Siebenmorgen, W. Freudling, E. Krügel, M. Haas, A\&A 421, 129 (2004).

[16] Y. Shi , G. H. Rieke, D. C. Hines et al., ApJ 629, 88 (2005).

[17] R. E. Spencer, J. C. McDowell, M. Charlesworth et al., MNRAS 240, 657 (1989). 